\title{
Sialólito de glândula salivar submandibular: relato de caso
}

\author{
Sialolith of submandibular salivary gland: case report
}

\author{
Alessandra Kuhn-Dall'Magro* \\ Caroline Carlos Alves ${ }^{* *}$ \\ Jonathan Lauxen ${ }^{* * *}$ \\ Renato dos Santos ${ }^{* * * *}$ \\ Túlio del Conte Valcanaia ${ }^{* * * *}$ \\ Eduardo Dall'Magro ${ }^{* * * * *}$
}

\section{Resumo}

Objetivo: ilustrar um caso de sialolitíase localizado na glândula submandibular. A sialolitíase está entre as enfermidades mais comuns das glândulas salivares. Apresenta crescimento lento, é uma patologia benigna, mas de etiologia incerta. A maioria dos casos ocorre no ducto de glândula submandibular, devido ao trajeto do ducto de Wharton que é sinuoso e ascendente, com maior facilidade para deposição dos sais de cálcio e consequente formação de cálculos, além da maior quantidade de proteínas secretadas pela glândula submandibular, o que torna a secreção mais espessa. O diagnóstico é feito por meio da avaliação intra e extraoral, além do auxílio de exames complementares, tais como radiografia, ultrassonografia, tomografia computadorizada (TC) e sialometria. O tratamento está intimamente ligado ao tamanho e à localização do cálculo e sempre se opta, quando possível, pelo mais conservador. Relato de caso: paciente do gênero feminino, leucoderma, 53 anos, portadora de um sialólito no ducto da glândula submandibular do lado esquerdo. Foi realizado tratamento cirúrgico, por acesso intraoral, na região do ducto obliterado, mantendo a glândula afetada em funcionamento. A paciente foi acompanhada por seis meses após a remoção do sialólito sem recidivas ou intercorrências pós-operatórias. Considerações finais: de acordo com o caso ilustrado neste trabalho e o que foi observado na literatura revisada, sialólito é uma calcificação que ocorre nos ductos das glândulas salivares, de origem idiopática, com tamanho e localização variados. Nesse sentido, o tratamento de escolha inicial para a remoção de sialólito deve ser conservador, optando-se por excisão cirúrgica quando o primeiro não for possível, e em casos extremos excisão da glândula afetada. O diagnóstico precoce é imprescindível devido à sintomatologia dolorosa e o desconforto.

Palavras-chave: Sialolitíase. Glândula submandibular. Sialólito.

\section{Introdução}

A sialolitíase é a presença de uma estrutura calcificada (sialólito) dentro dos ductos das glândulas salivares, ou menos frequentemente, dentro das glândulas. $\mathrm{O}$ sialólito, ou o cálculo (pedra), surge por meio de deposição de sais de cálcio ao redor de um ninho central, formando então a calcificação, sendo mais comum em glândulas submandibulares, mas

Especialista em Cirurgia e Traumatologia Bucomaxilofacial (CTBMF) na Santa Casa em Porto Alegre; Mestra em Ciências Médicas na Faculdade de Medicina na Universidade Federal do Rio Grande do Sul (UFRGS); professora dos cursos de Pós-Graduação em Cirurgia e Traumatologia Bucomaxilofacial do Centro de Estudos Odontológicos Meridional (Ceom) de Passo Fundo e do Instituto Odontológico das Américas (IOA) em Balneário Camboriú-SC; membro do corpo clínico do Hospital São Vicente de Paulo de Passo Fundo (HSVP) e do Instituto de Ortopedia e Traumatologia (IOT)em Passo Fundo,RS, Brasil.

* Cirurgiã-dentista graduada pela Faculdade de Odontologia da Universidade de Passo Fundo (FOUPF), RS, Brasil.

*** Cirurgião-dentista graduado pela Faculdade de Odontologia da Universidade de Passo Fundo (FOUPF), Passo Fundo, RS, Brasil.

**** Especialista em Cirurgia e Traumatologia Bucomaxilofacial (CTBMF)- CEOM - HSVP, Passo Fundo, RS, Brasil.

***** Especialista e Mestre em Cirurgia e Traumatologia Bucomaxilofacial (CTBMF), Universidade Federal de Pelotas (UFPEL); Doutor em Cirurgia e Traumatologia Bucomaxilofacial na Pontifícia Universidade Católica do Rio Grande do Sul (PUCRS); professor de Cirurgia e Traumatologia Bucomaxilofacial da Faculdade Avantis; coordenador do curso de Especialização em Implantodontia da Faculdade Avantis, Balneário Camboriú, SC, Brasil.

${ }^{* * * * * *}$ Especialista em Prótese Dentária pela Universidade de São Paulo (USP); Mestre em Laser em Odontologia pela Universidade de São Paulo (USP); Mestre e Doutor em Materiais Dentários pela Universidade Estadual de Campinas (Unicamp); professor titular III da Faculdade de Odontologia da Universidade de Passo Fundo (FOUPF), Passo Fundo, RS, Brasil. 
podendo ocorrer nas glândulas parótidas ou ainda em glândulas salivares menores e unilateralmente ${ }^{1}$.

Lagares et al. ${ }^{2}$ (2006) indicam que a sialolitíase é responsável por $30 \%$ das doenças das glândulas salivares, envolvendo mais comumente a glândula submandibular ( $83 \%$ a $94 \%$ ) e menos frequente as glândulas parótidas ( $4 \%$ a $10 \%)$ e sublingual (1\% a $7 \%$ ). O quadro clínico de sialolitíase é muito característico e facilmente diagnosticado especialmente pela sintomatologia dolorosa que ocorre em $83 \%$ dos casos.

Entre os métodos de diagnóstico da sialolitíase incluem-se a inspeção e a palpação, além da verificação da quantidade e da qualidade da saliva secretada. Durante as refeições, pela estimulação salivar, podem apresentar aumento de volume glandular e discreta sensação de desconforto, sendo a severidade da sintomatologia, quando presente, diretamente relacionada ao grau de obstrução do ducto ${ }^{3,4}$.

Várias causas sobre a formação desses cálculos são sugeridas pela literatura: mecânicas, químicas, inflamatórias, neurogênicas e infecciosas, causando precipitação do fosfato de tricálcio amorfo, o qual se cristaliza e se transforma em hidroxiapatita. São caracterizados pela presença de uma massa discreta, inflamação palpável ou difusa, podendo ser confundido com tumores (benignos ou malignos), adenopatias e hamartomas ${ }^{1-3}$.

A maioria $(80 \%)$ dos sialólitos apresentam-se radiopacos, sendo que o grau de radiopacidade depende de sua composição mineral. Podem ser facilmente observados em radiografias oclusais de mandíbula, embora exames auxiliares como tomografia computadorizada, ressonância magnética, ultrassonografia, sialoendoscopia e sialografia podem auxiliar no diagnóstico ${ }^{1-3}$.

Cobos et al. ${ }^{5}$ (2009) relataram as características histológicas dos sialólitos, por meio da microscopia como sendo material mineralizado de vários tons e densidade em torno de um núcleo mineral, na superfície são observados cálculos, fibras colágenas e células epiteliais metaplásicas.

Araújo et al. ${ }^{6}$ (2011) afirmam que a maior frequência de sialolitíase em glândula salivar submandibular é devido ao trajeto sinuoso e ascendente do ducto de Wharton, com maior facilidade para deposição dos sais de cálcio e consequente formação de cálculos, além da maior quantidade de proteínas (mucinas) secretadas pela glândula submandibular, tornando a secreção mais espessa, dificultando a drenagem, aumentando a concentração de cálcio na saliva e constituindo mais um fator predisponente à obliteração do ducto. Acredita-se que a origem dos cálculos está relacionada à deposição de sais de cálcio ao redor de bactérias, células epiteliais do ducto ou dos corpos estranhos, sendo a porção central predominantemente inorgânica e constituída, em sua maioria, de diferentes formas de fosfato de cálcio com quantidades variadas de carbonatos na forma de hidroxiapatita, podendo conter também magnésio, potássio e amônia, já, em sua periferia contém componentes inorgânicos e orgânicos, sendo esses: glicoproteínas, mucopolissacarídeos, lipídios e detritos celulares.

Augusto et al. ${ }^{7}$ (2011) indicam que os sialólitos ficam localizados nos dois terços anteriores do ducto de Wharton, são facilmente palpados intraoralmente e detectados na radiografia oclusal.

Chuangqi et al. ${ }^{8}$ (2013) fizeram estudos sobre glândulas submandibulares e parótidas em pacientes, cuja queixa principal era o inchaço. Utilizaram a sialodenoscopia para conseguir informação sobre os ductos salivares, e em alguns casos indicar a possível obstrução. Encontraram como patologia principal a presença de sialólitos.

O objetivo deste estudo é ilustrar, por meio de caso clínico, o tratamento cirúrgico de um sialólito de aproximadamente $15 \mathrm{~mm}$ localizado no ducto da glândula submandibular esquerda.

\section{Relato de caso}

Paciente do gênero feminino, 53 anos, procurou o Ambulatório de Especialidades do Hospital São Vicente de Paulo de Passo Fundo, na Especialidade de Cirurgia e Traumatologia Bucomaxilofacial, com queixas de sintomatologia dolorosa ao se alimentar, bem como tumefação na região esquerda da mandíbula, e ainda presença de assimetria facial (Figura 1A). Ao exame de palpação intraoral, apresentou nódulo no assoalho bucal do lado esquerdo (Figura 1B). Após realização de exames clínicos, foi realizado exame radiográfico oclusal de mandíbula, o que evidenciou uma calcificação adjacente ao corpo mandibular (Figura 2). Para melhor elucidação radiográfica realizou-se a TC, sendo identificada uma massa hiperdensa, radiopaca, lingualizada e superior em relação ao rebordo alveolar do lado esquerdo relacionada à glândula submandibular (Figura 3). Após preparos pré-cirúrgicos, como assepsia extra e intraoral, foi realizada a anestesia (Figura 4), o acesso intraoral na região do ducto foi obliterado com eletrocautério, a divulsão tecidual até a localização da lesão (Figura 5A e 5B), exérese total dessa (Figura 6A) e sutura com fio vicryl 4.0 (Figura 6B).

O sialólito removido media aproximadamente $15 \mathrm{~mm}$ em sua maior extensão (Figura 7). Houve a manutenção da glândula afetada. $\mathrm{O}$ exame anatomopatológico confirmou a hipótese de sialólito. A paciente foi acompanhada por seis meses sem recidiva da lesão, não ocorrendo nenhum tipo de intercorrências tanto no transoperatório, quanto no pós-operatório. 

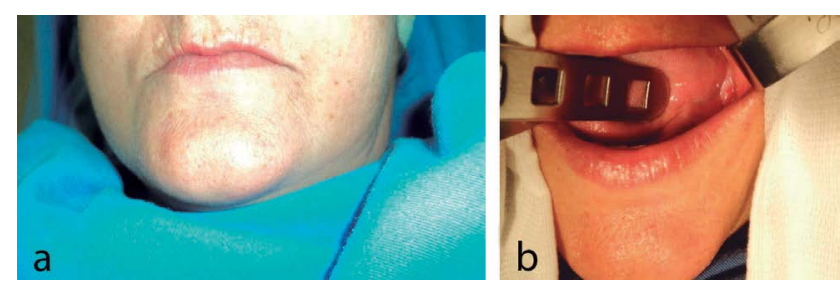

Fonte: acervo da autora Dra. Alessandra Kuhn-Dall'Magro.

Figura 1 - a) Assimetria da face do lado esquerdo; b) Aspecto intraoral do sialólito

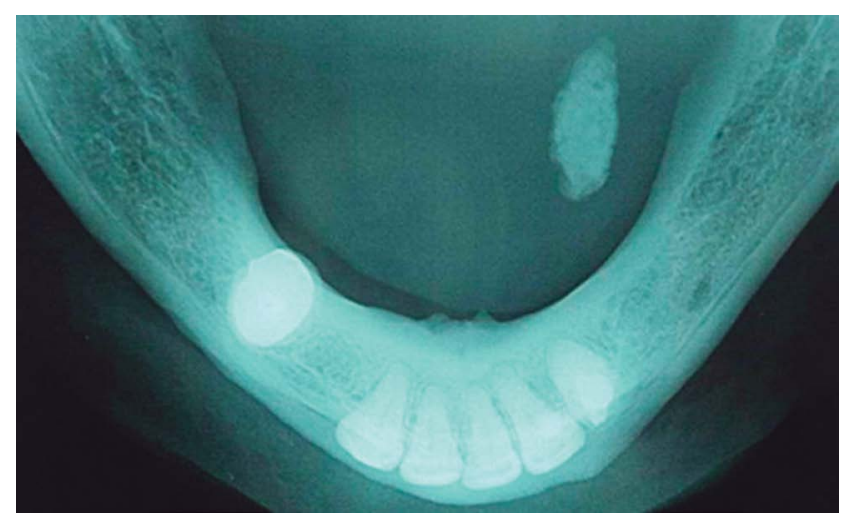

Fonte: acervo da autora Dra. Alessandra Kuhn-Dall’Magro.

Figura 2 - Exame radiográfico oclusal

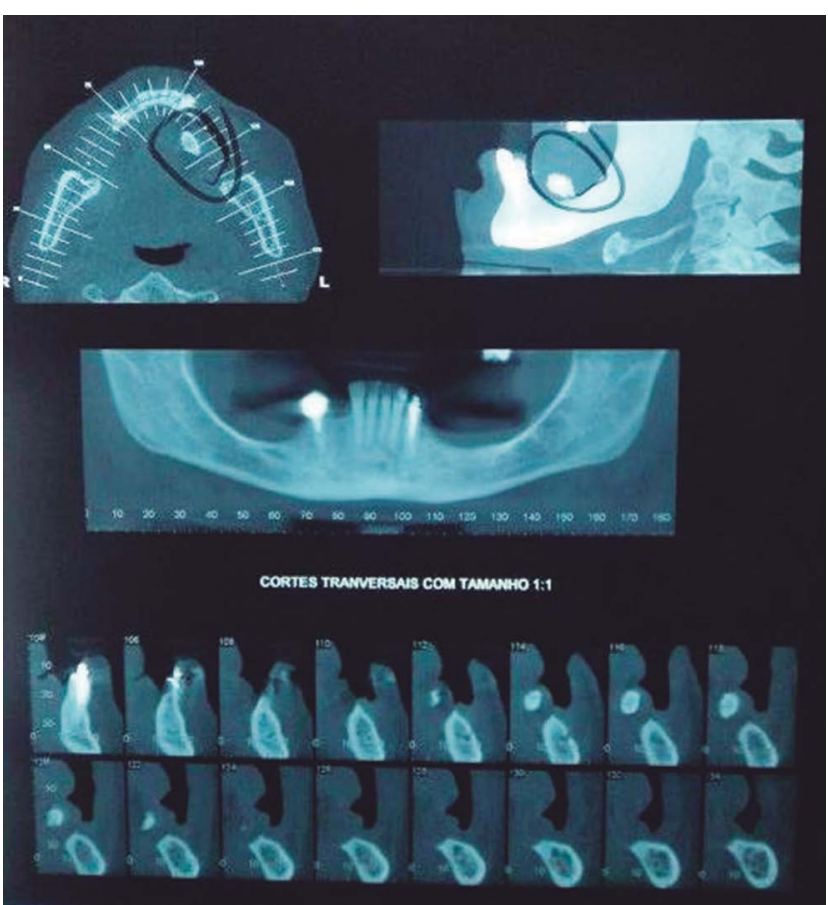

Fonte: acervo da autora Dra. Alessandra Kuhn-Dall'Magro.

Figura 3 - Tomografia computadorizada

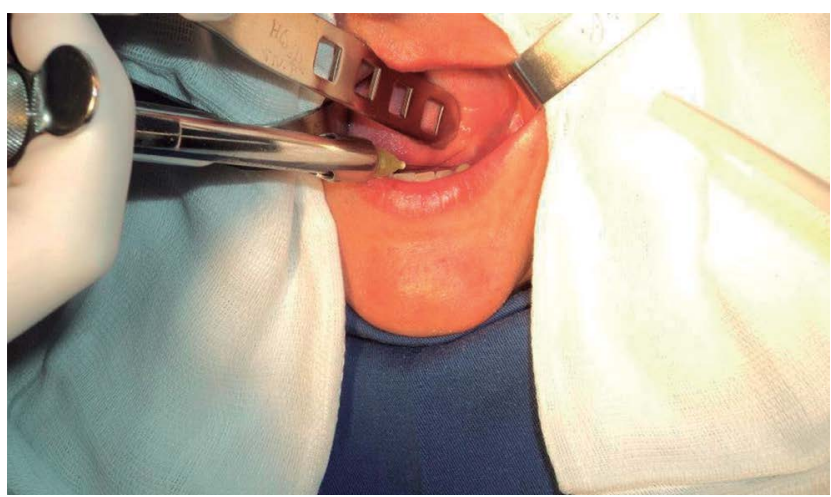

Fonte: acervo da autora Dra. Alessandra Kuhn-Dall'Magro.

Figura 4 - Técnica anestésica

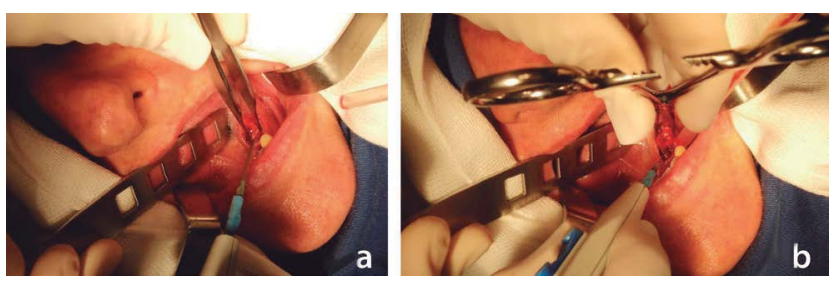

Fonte: acervo da autora Dra. Alessandra Kuhn-Dall’Magro.

Figura 5 - a) Incisão com eletrocautério; b) Divulsão tecidual e exérese do sialólito
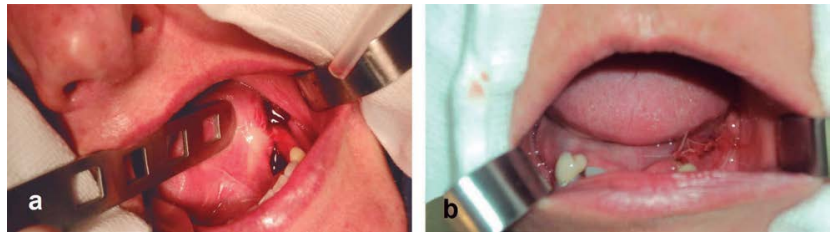

Fonte: acervo da autora Dra. Alessandra Kuhn-Dall’Magro.

Figura 6 - a) Imagem logo após remoção do sialólito; b) Sutura (fio vicryl 4.0)
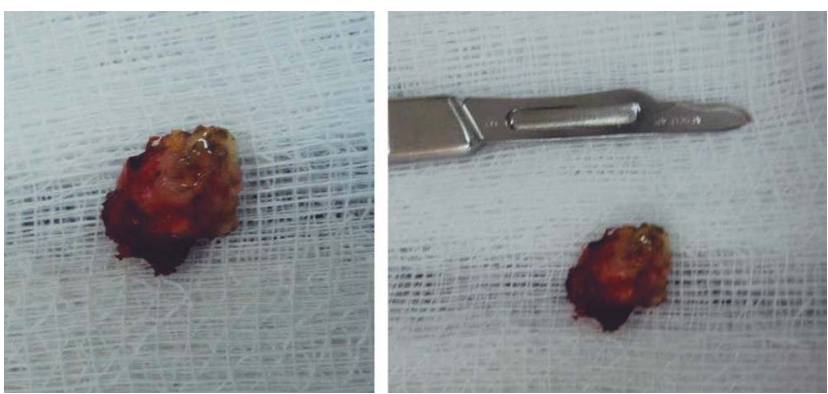

Fonte: acervo da autora Dra. Alessandra Kuhn-Dall'Magro.

Figura 7 - Sialólito medindo aproximadamente $15 \mathrm{~mm} \times 10 \mathrm{~mm}$

\section{Discussão}

A sialolitíase é a presença de uma estrutura calcificada (sialólito) dentro dos ductos das glândulas salivares, ou menos frequentemente, dentro das glândulas ${ }^{1}$. É responsável por 30\% das doenças salivares e mais comumente envolve a glândula submandibular ( $83 \%$ a $94 \%$ ) e menos frequente as glândulas parótidas (4\% a $10 \%)$ e sublingual (1\% a $7 \%$ ). Embora o presente trabalho relate um caso de sialólito em uma paciente do gênero feminino, 
ocorre com maior prevalência no gênero masculino especialmente na raça branca.

Manzi et al. ${ }^{9}$ (2010) dividem a etiologia do sialólito em dois grupos: primeiramente, pela retenção da saliva, devido a fatores morfo-anatômicos (esteanose do tubo salivar ou diverticulação de tubo salivar); e o fator da composição salivar (saturação alta, cristalização ou déficit de inibidores). Já Soares et al. ${ }^{10}$ (2010) argumentam que mesmo tendo causa específica desconhecida, originam-se da deposição de sais de cálcio ao redor de acúmulo de restos orgânicos no lúmen do ducto, constituídos de muco condensado, bactérias, células epiteliais do ducto ou corpos estranhos, mas afirmam que sua produção pode ser provocada por sialodenite crônica e obstrução parcial. Sendo o sialólito um evento multifatorial, a disfunção salivar pode ocorrer a partir de doenças sistêmicas ou medicamentos, os distúrbios secretores podem influenciar na sua formação, pois um dos componentes da saliva, a glicoproteína, tem afinidade elevada de cálcio, que contribui para a mineralização da matriz orgânica, sendo essa reforçada pelo acúmulo de cálcio e diminuição no $\mathrm{pH}$ que, por sua vez, diminui a solubilidade dos fosfatos de cálcio na saliva.

Huoh e Eisele ${ }^{11}$ (2011) estudaram um grupo de pacientes portadores de sialólitos e acreditam na possível associação com o hábito de fumar, bem como uma taxa de aumento de sialólitos por uso de diuréticos, embora sugiram pesquisas mais avançadas para elucidar os riscos relativos ao fumo e a outros fatores que podem contribuir para sialolitíase. Também foi encontrado, na literatura revisada, um caso incomum como o relatado por Boynton e Liebli$\operatorname{ch}^{12}$ (2014), no qual a barba do paciente aparentemente ficou retida no ducto, originando a formação do sialólito.

A paciente estudada relatou dor ao se alimentar e tumefação na região esquerda da mandíbula, coincidindo com a principal queixa dos outros casos relatados encontrados na literatura. Kawata et al. ${ }^{4}$ (2006) descreveram dois casos clínicos de sialólitos: no primeiro, além da sintomatologia dolorosa, o paciente apresentava uma massa endurecida à palpação, e a presença intrabucal de secreção purulenta, drenada por meio da carúncula direita e palpando ao longo do trajeto do ducto salivar identificaram uma massa endurecida submucosa com aproximadamente $2 \mathrm{~cm}$ de diâmetro. Já no segundo caso, o paciente apresentou nódulo submucoso intrabucal com aproximadamente $0,5 \mathrm{~cm}$ e ausência de sintomatologia dolorosa. Os casos encontrados na literatura mostram tamanhos variados dos sialólitos. Ramos-Jorge et al. ${ }^{13}$ (2006) acreditam que sialólitos maiores que $15 \mathrm{~mm}$ são considerados raros e existem poucos casos descritos, têm crescimento lento, e a sintomatologia dolorosa, quando presente, está diretamente ligada a obstrução do ducto salivar. No caso relatado neste trabalho, a paciente apresentava sialólito com aproximadamente $15 \mathrm{~mm}$, tamanho considerado raro pela literatura, e intensa sintomatologia dolorosa.

Cálculos menos profundos podem ser removidos com simples manipulação, mas o mais comum é a remoção cirúrgica por acesso intraoral ${ }^{1,14-16}$. Métodos mais sofisticados como a sialoendoscopia, a cirurgia a laser e a litotripsia têm sido sugeridos pela literatura como alternativas de tratamento ${ }^{16,17}$. A remoção das glândulas envolvidas em processo de sialolitíase é indicada quando se torna sintomática, envolvendo tumefação, inflamação e fibrose dessa, causando dor e tumefação persistente ${ }^{1}$. Como tratamento não invasivo antes da cirurgia, pode-se indicar fisioterapia com fontes de calor externo e interno, bochechos com clorexidina e água com limão, e massagens, embora o prognóstico de sucesso com tais técnicas esteja relacionado ao tamanho e à posição do sialólito. Alguns autores ${ }^{14}$ defendem a indicação cirúrgica para a remoção de sialólitos apenas quando esses se localizarem na porção distal dos ductos, sendo anterior ao primeiro molar inferior em relação ao ducto de Wharton, visto que a tentativa de remoção cirúrgica, além desse limite representa um elevado risco de lesão às estruturas nobres, como o nervo lingual e hipoglosso, sendo indicada para essa finalidade a remoção via endoscópica, o que reduziria substancialmente essa possibilidade. No entanto, estudo realizado por Juul e Wagner ${ }^{16}$, envolvendo 42 pacientes com sialólitos removidos cirurgicamente por acesso intraoral indicou $94 \%$ de sucesso com tal técnica, $3 \%$ de dano ao nervo lingual e $6 \%$ de formigamento lingual.

\section{Considerações finais}

De acordo com o caso ilustrado nesta pesquisa e o que foi observado na literatura revisada, sialólito é uma calcificação que ocorre nos ductos das glândulas salivares, de origem idiopática, com tamanho e localização variados. Nesse sentido, o tratamento de escolha inicial para remoção de sialólito deve ser conservador, optando-se por excisão cirúrgica quando o primeiro não for possível, e em casos extremos de excisão da glândula afetada. O diagnóstico precoce é imprescindível devido à sintomatologia dolorosa e o desconforto.

\section{Abstract}

Objective: this article aims to illustrate a case of sialolithiasis in the submandibular gland. Sialolithiasis is among the most common diseases of salivary glands. It is a slow-growing benign pathology, but of uncertain etiology. Most cases occur in the submandibular gland duct, due to the route of Wharton's duct, which is winding and ascending, with easier deposition of calcium salts and consequent stone formation, besides the greater amount of proteins secreted by the submandibular gland, which makes secretion thicker. Diagnosis is 
determined by intra- and extraoral assessment, and by the aid of complementary exams such as radiographs, ultrasound, computed tomography (CT), and sialometry. The treatment is closely linked to the size and location of the stone, and whenever possible, the most conservative treatment is chosen. Case Report: female patient, leucoderma, 53 years old, carries a sialolith in the duct of the left submandibular gland. Surgical treatment was performed intraorally into the obliterated duct, keeping the affected gland functioning. The patient was followed for six months after the removal of the sialolith with no postoperative recurrences or complications. Final considerations: according to the case illustrated in this study and the findings of literature review, sialolith is a calcification that occurs in salivary gland ducts, from idiopathic origin, with varying sizes and locations. Therefore, the initial choice of treatment for removing the sialolith should be the conservative one, opting for surgical excision when the first choice of treatment is not feasible, and in extreme cases of excision of the affected gland. Early diagnosis is essential due to painful symptomatology and discomfort.

Keywords: Sialolithiasis. Submandibular gland. Sialolith.

\section{Referências}

1. Neville BW, Damm DD, White DK. Atlas colorido de patologia oral clínica. 2. ed. Rio de Janeiro: Guanabara Koogan; 2001. $486 \mathrm{p}$.

2. Lagares DT, Pietra SB, Figatto MAS, Iglesias PH, Márquez AMS, Pérez JLG. Sialolitiasis parotídea el conducto de stensen, med oral patol cir bucal 2006; 11: E80-E84; 2006.

3. Melo AR, Santos TS, Amaral MF. Técnica cirúrgica para remoção intrabucal de sialólito. Rev Cir Traumatol Buco-Maxilo-Fac 2011;11(4):55-8.

4. Kawata LT, Baldan RCF, Moraes NP, Biazolla ER. Sialolitíase em ducto de glândula submandibular: relato de dois casos clínicos. Rev Odontol Araçatuba 2006; 27(1):14-8.

5. Cobos RM, Carbonell ZM, Knight DA. Sialólitos em conductos y glândulas salivales. Revisión de literatura, Avances em Odontoestomatología 2009; 25(6):311-7.

6. Araújo FAC, Farias ON, Landim FS, Fernandez AV, Caubil AF. Tratamento cirúrgico de sialólito de glândula submandibular: relato de caso. Rev Cir Traumatol Buco-Maxilo-Fac. $2011 ; 11(4): 13-8$.

7. Augusto G, Pontes MA, Coletti JM, Ribeiro AO, Souza LMA. Contribuição ao estudo dos sialólitos: relato de caso. Rev. Port. Estomatol. Med. Dent. Cir. Maxilofac 2011; 52(3):161-4.

8. Chuangqi Y, Chi Y, Lingyan Z. Sialendoscopic findings in patients with obstructive sialadenitis: long-term experience. $\mathrm{Br}$ J Oral Maxillofac Surg 2013; 51(4):337-41.

9. Manzi FR, Silva AIV, Dias FG, Ferreira EF. Sialólito na glândula submandibular: relato de caso clínico. Rev Odontol Bras Central 2010; 19(50):270-4.

10. Soares GS, Silva ARS, Soubhia AMP, Miyahara GI. Considerações atuais da sialolitíase de ducto de glândula submandibular. Rev Odontol de Araçatuba 2010; 31(1):46-50.

11. Huoh KC, Eisele DW. Etiologic factors in sialolithiasis. otolaryngol head neck surg. 2011; 145(6):935-9.

12. Boynton TT, Lieblich SF. Unusual case of a sialolith: a case report. oral surg oral med oral pathol oral radiol 2014; 117(1):9-10.
13. Ramos-Jorge J, Rego TM, Santos CRR. Sialolitíase em glândula submandibular: relato de caso clínico. Arquivos em Odontologia 2006; 42:81-160.

14. Potash A, Hoffman HT. Retrograde sialendoscopy: a new technique for avoiding retained ductal stones. Ann otol rhinol laryngol 2012;121(1):38-43.

15. Parkar MI, Vora MM, Bhanushali DH. A large sialolith perforating the Wharton's duct: review of literature and a case report. J Maxillofac Oral Surg 2012;11(4):477-82.

16. Juul ML,Wagner N. Objective and subjective outcome in 42 patients after treatment of sialolithiasis by transoral incision of Warthon's duct: a retrospective middle-term follow-up study. Eur Arch Otorhinolaryngol 2014 Feb 4. [Epub ahead of print]

17. Klein H, Ardekian L. The treatment of large sialoliths by sialendoscopic combined approach. J Oral Maxillofac Surg 2014; 72(4):737-43.

\section{Endereço para correspondência:}

Alessandra Kuhn-Dall'Magro

Teixeira Soares, no777/02 - Centro

99010-080 Passo Fundo-RS

Fone: (54) 36010723

E-mail: alessandrakuhn@hotmail.com

Recebido: 23/03/2014. Aceito: 23/10/2014. 